\title{
On a generalization of the Selberg trace formula
}

\author{
by
}

\section{A. Biró (Budapest)}

1. Introduction. The Selberg trace formula (the original paper is [Se]; for a nice account see [I]) is obtained (e.g. for a co-compact Fuchsian group $\Gamma$ with fundamental domain $F$ in $H$, where $H$ is the upper half-plane) by computing in two different ways (geometrically and spectrally) the integral

$$
\operatorname{Tr} K=\int_{F} K(z, z) d \mu_{z},
$$

where $K(z, w)$ is an automorphic kernel function. We take here instead of $\operatorname{Tr} K$ an integral of the form

$$
\operatorname{Tr}_{u} K=\int_{F} K(z, z) u(z) d \mu_{z}
$$

where $u$ is an automorphic eigenfunction of the Laplace operator, so we write $u(z)$ in place of the identically 1 function.

On the geometric side of our formula we get integrals of $u$ on certain closed geodesics of the Riemann surface $\Gamma \backslash H$. On the spectral side integrals of the form

$$
\int_{F}\left|u_{j}(z)\right|^{2} u(z) d \mu_{z}
$$

appear (the $u_{j}$ run over an orthonormal basis of automorphic Laplaceeigenforms), so our formula (Theorem 1) is a duality between such integrals and certain geodesic integrals of $u$. New integral transformations are involved depending on the Laplace-eigenvalue of $u$. We invert these integral transformations in Section 5, Theorem 2.

We develop the formula for finite volume Fuchsian groups, so (as in the case of the Selberg trace formula) $\int_{F} K(z, z) u(z) d \mu_{z}$ will not be convergent, and we take instead

$$
\operatorname{Tr}_{u}^{Y} K=\int_{F(Y)} K(z, z) u(z) d \mu_{z},
$$

1991 Mathematics Subject Classification: Primary 11F72. 
where $F(Y)$ is obtained from $F$ by cutting off the cuspidal zones at height $Y$. We let $Y \rightarrow \infty$, and the main term (which is in our case a power of $Y$, while in the case of the Selberg trace formula the main term is $\log Y$ ) will cancel out. An interesting feature of our formula is the appearance of the Riemann zeta-function in the contribution of the parabolic conjugacy classes.

In Section 6 we prove lemmas on special functions needed in Section 5.

Acknowledgements. I would like to thank Professor G. Halász for introducing me to the Selberg trace formula.

2. Notations and statement of the main result. Let $H$ be the open upper half-plane. The elements $\left(\begin{array}{ll}a & b \\ c & d\end{array}\right)$ of the group $\operatorname{PSL}(2, \mathbb{R})$ act on $H$ by the rule $z \rightarrow(a z+b) /(c z+d)$. The hyperbolic Laplace operator is given by

$$
\Delta=y^{2}\left(\frac{\partial^{2}}{\partial x^{2}}+\frac{\partial^{2}}{\partial y^{2}}\right) \text {. }
$$

It is well known that $\Delta$ commutes with the action of $\operatorname{PSL}(2, \mathbb{R})$.

Let $\Gamma \subset \operatorname{PSL}(2, \mathbb{R})$ be a finite volume Fuchsian group, i.e. $\Gamma$ acts discontinuously on $H$, and it has a fundamental domain of finite volume.

The constants in the symbols $O$ will depend on the group $\Gamma$. For a function $f$ we denote its $j$ th derivative by $f^{(j)}$.

We fix a complete set $A$ of inequivalent cusps of $\Gamma$, and we will denote the elements of $A$ by $a, b$ or $c$, so e.g. $\sum_{a} \sum_{c}$ or $\bigcup_{a}$ will mean that $a$ and $c$ run over $A$. We say that $\sigma_{a}$ is a scaling matrix of a cusp $a$ if $\sigma_{a} \infty=a$, $\sigma_{a}^{-1} \Gamma_{a} \sigma_{a}=B$, where $\Gamma_{a}$ is the stability group of $a$ in $\Gamma$, and $B$ is the group of integer translations. The scaling matrix is determined up to composition with a translation from the right.

We also fix a complete set $P$ of representatives of $\Gamma$-equivalence classes of the set $\{z \in H: \gamma z=z$ for some id $\neq \gamma \in \Gamma\}$. For a $p \in P$ let $m_{p}$ be the order of the stability group of $p$ in $\Gamma$.

Let

$$
P(Y)=\{z=x+i y: 0<x \leq 1, y>Y\},
$$

and let $Y_{\Gamma}$ be a constant (depending only on the group $\Gamma$ ) such that for any fixed $Y \geq Y_{\Gamma}$ the cuspidal zones $F_{a}(Y)=\sigma_{a} P(Y)$ are disjoint, and the fixed fundamental domain $F$ of $\Gamma$ (it contains exactly one point of each $\Gamma$-equivalence class of $H$ ) is partitioned into

$$
F=F(Y) \cup \bigcup_{a} F_{a}(Y)
$$

where $F(Y)$ is the central part,

$$
F(Y)=F \backslash \bigcup_{a} F_{a}(Y),
$$

and $F(Y)$ has compact closure. 
Denote by $\left\{u_{j}(z): j \geq 0\right\}$ a complete orthonormal system of Maass forms for $\Gamma$ for the discrete spectrum $\left(u_{0}(z)\right.$ is constant), with Laplace-eigenvalue $\lambda_{j}=s_{j}\left(s_{j}-1\right), \operatorname{Re} s_{j} \geq 1 / 2, s_{j}=1 / 2+i t_{j}$ and Fourier expansion

$$
u_{j}\left(\sigma_{a} z\right)=\beta_{a, j}(0) y^{1-s_{j}}+\sum_{n \neq 0} \beta_{a, j}(n) W_{s_{j}}(n z),
$$

where $W$ is the Whittaker function.

The Fourier expansion of the Eisenstein series (as in $[\mathrm{I}],(8.2)$ ) is given by

$$
\begin{aligned}
E_{c}\left(\sigma_{a} z, 1 / 2+i r\right)= & \delta_{a c} y^{1 / 2+i r}+\varphi_{a, c}(1 / 2+i r) y^{1 / 2-i r} \\
& +\sum_{n \neq 0} \varphi_{a, c}(n, 1 / 2+i r) W_{1 / 2+i r}(n z) .
\end{aligned}
$$

Let $\left\{s_{l}: l \in L\right\}$ be the set of the poles of the Eisenstein series for $\Gamma$. Then $1 / 2<s_{l} \leq 1$ for every $l \in L$, and $L$ is a finite set. We have $\beta_{a, j}(0)=0$ if $j>0$, and $u_{j}(z)$ is not a linear combination of residues of Eisenstein series, so if $j>0$ is such that $\beta_{a, j}(0) \neq 0$ for some $a$, then $s_{j}=s_{l}$ for some $l \in L$. The functions $\varphi_{a, a}(s)$ may have poles only at the points $s_{l}$. Let us denote the residue of $\varphi_{a, a}(s)$ at $s=s_{l}$ by $R_{a, s_{l}}$, when $l \in L$.

Let $1 / 2 \leq \operatorname{Re} s<1$, and let $u(z)$ be a fixed $\Gamma$-automorphic eigenfunction of the Laplace operator with eigenvalue $\lambda=s(s-1)$, and Fourier expansion at any cusp $a$ of $\Gamma$

$$
u\left(\sigma_{a} z\right)=\beta_{a}(0) y^{s}+\widetilde{\beta}_{a}(0) y^{1-s}+\sum_{n \neq 0} \beta_{a}(n) W_{s}(n z) .
$$

For simplicity we assume that $s \neq 2 s_{l}-1$ for $l \in L$.

We introduce the notations

$$
\begin{gathered}
B_{u}=\sum_{a} \beta_{a}(0), \quad \widetilde{B}_{u}=\sum_{a} \widetilde{\beta}_{a}(0), \\
B_{u}(S)=\sum_{a} \beta_{a}(0) \varphi_{a, a}\left(\frac{1+S}{2}\right),
\end{gathered}
$$

Let $k$ be a function on $[0, \infty)$, and assume that it satisfies

(A) $\quad k$ is a compactly supported continuous function on $[0, \infty)$.

As usual (see [I], (1.62)), let

$$
g(a)=2 q\left(\frac{e^{a}+e^{-a}-2}{4}\right), \text { where } q(\nu)=\int_{0}^{\infty} \frac{k(\nu+\tau)}{\sqrt{\tau}} d \tau,
$$

and let $h$ be the Fourier transform of $g$,

$$
h(r)=\int_{-\infty}^{\infty} g(a) e^{i r a} d a .
$$


We assume that

(B) $\quad h(r)$ is even, it is holomorphic in the strip $|\operatorname{Im} r| \leq 1 / 2+\varepsilon$, and $h(r)=O\left((1+|r|)^{-2-\varepsilon}\right)$ in this strip for some $\varepsilon>0$.

The point-pair invariant determined by $k$ is

$$
k(z, w)=k\left(\frac{|z-w|^{2}}{4 \operatorname{Im} z \operatorname{Im} w}\right)
$$

for $z, w \in H$. The automorphic kernel function $K(z, w)$ is given by

$$
K(z, w)=\sum_{\gamma \in \Gamma} k(z, \gamma w) .
$$

Define

$$
\operatorname{Tr}_{u}^{Y} K=\int_{F(Y)} K(z, z) u(z) d \mu_{z} .
$$

We will determine the asymptotic behaviour of $\operatorname{Tr}_{u}^{Y} K$ as $Y \rightarrow \infty$ in two different ways. Firstly, by partitioning $\Gamma$ into conjugacy classes, and secondly, using the spectral theorem (which is applicable by our conditions on $k$ and $h$ ), since introducing the notations

$I_{u}^{Y}(r)=\sum_{c} \int_{F(Y)}\left|E_{c}(z, 1 / 2+i r)\right|^{2} u(z) d \mu_{z}, \quad I_{u}^{Y}\left(u_{j}\right)=\int_{F(Y)}\left|u_{j}(z)\right|^{2} u(z) d \mu_{z}$,

we have by the spectral theorem

$$
\operatorname{Tr}_{u}^{Y} K=\sum_{j} h\left(t_{j}\right) I_{u}^{Y}\left(u_{j}\right)+\frac{1}{4 \pi} \int_{-\infty}^{\infty} h(r) I_{u}^{Y}(r) d r .
$$

We give here the statement of our Lemma 4 (which will be proved in Section 4 below), because to state Theorem 1 we need the quantities $I_{u}\left(u_{j}\right)$ and $I_{u}(r)$ defined in that lemma.

Lemma 4. Define

$$
\begin{aligned}
\psi_{a}^{Y}(r, s)= & \frac{Y^{s}}{s}+\varphi_{a, a}(1 / 2+i r) \frac{Y^{s-2 i r}}{s-2 i r}, \\
\widetilde{I}_{u}^{Y}(r)= & I_{u}^{Y}(r)-\left(\sum_{a} \beta_{a}(0)\left(\psi_{a}^{Y}(r, s)+\psi_{a}^{Y}(-r, s)\right)\right. \\
& \left.+\widetilde{\beta}_{a}(0)\left(\psi_{a}^{Y}(r, 1-s)+\psi_{a}^{Y}(-r, 1-s)\right)\right),
\end{aligned}
$$

and

$$
\widetilde{I}_{u}^{Y}\left(u_{j}\right)=I_{u}^{Y}\left(u_{j}\right)-\sum_{a}\left|\beta_{a, j}(0)\right|^{2}\left(\beta_{a}(0) \frac{Y^{1+s-2 s_{j}}}{1+s-2 s_{j}}+\widetilde{\beta}_{a}(0) \frac{Y^{2-s-2 s_{j}}}{2-s-2 s_{j}}\right) .
$$


Then

$$
\sum_{\left|t_{j}\right| \leq R}\left|\widetilde{I}_{u}^{Y}\left(u_{j}\right)\right|+\int_{-R}^{R}\left|\widetilde{I}_{u}^{Y}(r)\right| d r=O\left(R^{2}\right)
$$

uniformly in $Y$. The limits

$$
I_{u}(r)=\lim _{Y \rightarrow \infty} \widetilde{I}_{u}^{Y}(r), \quad I_{u}\left(u_{j}\right)=\lim _{Y \rightarrow \infty} \widetilde{I}_{u}^{Y}\left(u_{j}\right)
$$

obviously exist, and then, of course,

$$
\sum_{\left|t_{j}\right| \leq R}\left|I_{u}\left(u_{j}\right)\right|+\int_{-R}^{R}\left|I_{u}(r)\right| d r=O\left(R^{2}\right) .
$$

If $u_{j}(z)$ is not a linear combination of residues of Eisenstein series, then

$$
I_{u}\left(u_{j}\right)=\int_{F}\left|u_{j}(z)\right|^{2} u(z) d \mu_{z} .
$$

In particular $I_{u}\left(u_{0}\right)=0$.

With the above notations and assumptions our formula is the following:

TheOREM 1. Assume that $k$ satisfies condition (A) and $h$ satisfies condition (B). Let

$$
\Sigma_{\text {hyp }}=\sum_{\substack{[\gamma] \\ \gamma \text { hyperbolic }}}\left(\int_{C_{\gamma}} u d S\right) \int_{-\pi / 2}^{\pi / 2} k\left(\frac{N(\gamma)+N(\gamma)^{-1}-2}{4 \cos ^{2} \vartheta}\right) f_{\lambda}(\vartheta) \frac{d \vartheta}{\cos ^{2} \vartheta},
$$

where the summation is over the hyperbolic conjugacy classes of $\Gamma, N(\gamma)$ is the norm of (the conjugacy class of) $\gamma, C_{\gamma}$ is the closed geodesic obtained by factorizing the noneuclidean line connecting the fixed points of $\gamma$ by the action of the centralizer of $\gamma$ in $\Gamma, d S=|d z| / y$ is the hyperbolic arc length, and $f_{\lambda}(\vartheta)$ is the solution of the differential equation

$$
f^{(2)}(\vartheta)=\frac{\lambda}{\cos ^{2} \vartheta} f(\vartheta), \quad \vartheta \in(-\pi / 2, \pi / 2),
$$

with $f_{\lambda}(0)=1, f_{\lambda}^{(1)}(0)=0$. Let

$$
\Sigma_{\text {ell }}=\sum_{p \in P} \frac{2 \pi}{m_{p}} u(p) \sum_{l=1}^{m_{p}-1} \int_{0}^{\infty} k\left(\sin ^{2} \frac{l \pi}{m_{p}} \sinh ^{2} r\right) g_{\lambda}(r) \sinh r d r,
$$

where $g_{\lambda}(r)(r \in[0, \infty))$ is the unique solution of

$$
g^{(2)}(r)+\frac{\cosh r}{\sinh r} g^{(1)}(r)=\lambda g(r)
$$


with $g_{\lambda}(0)=1$. Let

$$
\Sigma_{\mathrm{par}}=B_{u} 2^{1-s} \zeta(1-s) \int_{0}^{\infty} k(\nu) \nu^{-(1+s) / 2} d \nu+\widetilde{B}_{u} 2^{s} \zeta(s) \int_{0}^{\infty} k(\nu) \nu^{(s-2) / 2} d \nu,
$$

where $\zeta$ is the Riemann zeta-function. Then the equality

$$
\begin{aligned}
\Sigma_{\text {hyp }}+\Sigma_{\text {ell }}+\Sigma_{\text {par }}= & \frac{1}{2} h\left(i \frac{s}{2}\right) B_{u}(s)+\frac{1}{2} h\left(i \frac{1-s}{2}\right) \widetilde{B}_{u}(1-s) \\
& +\sum_{j>0} h\left(t_{j}\right) I_{u}\left(u_{j}\right)+\frac{1}{4 \pi} \int_{-\infty}^{\infty} h(r) I_{u}(r) d r
\end{aligned}
$$

holds, where $I_{u}\left(u_{j}\right)$ and $I_{u}(r)$ is given in Lemma 4.

3. The geometric trace. For the first computation of $\operatorname{Tr}_{u}^{Y} K$ we partition $\Gamma$ into conjugacy classes $[\gamma]=\left\{\tau^{-1} \gamma \tau: \tau \in \Gamma\right\}$. Let id $\neq \gamma \in \Gamma$, and

$$
T_{\gamma}^{Y}=\sum_{\delta \in[\gamma]} \int_{F(Y)} k(z, \delta z) u(z) d \mu_{z}
$$

We have $\tau_{1}^{-1} \gamma \tau_{1}=\tau_{2}^{-1} \gamma \tau_{2}$ if and only if $\tau_{2} \tau_{1}^{-1} \in C(\gamma)$, where $C(\gamma)$ is the centralizer of $\gamma$ in $\Gamma$. So

$$
T_{\gamma}^{Y}=\sum_{\tau \in C(\gamma) \backslash \Gamma} \int_{F(Y)} k\left(z, \tau^{-1} \gamma \tau z\right) u(z) d \mu_{z} .
$$

Since $k\left(z, \tau^{-1} \gamma \tau z\right)=k(\tau z, \gamma \tau z)$ and $u(z)=u(\tau z)$, we obtain

$$
T_{\gamma}^{Y}=\int_{C(\gamma) \backslash H(Y)} k(z, \gamma z) u(z) d \mu_{z},
$$

where $H(Y)=\bigcup_{\gamma \in \Gamma} \gamma F(Y)$. Let $h \in \mathrm{SL}(2, \mathbb{R})$. Then

$$
\begin{aligned}
T_{\gamma}^{Y} & =\int_{h^{-1}(C(\gamma) \backslash H(Y))} k(h z, \gamma h z) u(h z) d \mu_{z} \\
& =\int_{\left(h^{-1} C(\gamma) h\right) \backslash\left(h^{-1} H(Y)\right)} k\left(z, h^{-1} \gamma h z\right) u(h z) d \mu_{z} .
\end{aligned}
$$

So far this is valid for every id $\neq \gamma \in \Gamma$. We now examine separately the case of hyperbolic, elliptic or parabolic transformations.

If $\gamma$ is hyperbolic or elliptic, then $T_{\gamma}=\lim _{Y \rightarrow \infty} T_{\gamma}^{Y}$ exists, and by (1) we get

$$
T_{\gamma}=\int_{\left(h^{-1} C(\gamma) h\right) \backslash H} k\left(z, h^{-1} \gamma h z\right) u(h z) d \mu_{z} .
$$

If $\gamma$ is hyperbolic, then we choose $h \in \mathrm{SL}(2, \mathbb{R})$ so that $h^{-1} \gamma h$ is a dilation, i.e. $h^{-1} \gamma h z=N(\gamma) z$ for $z \in H$, where $N(\gamma)>1(N(\gamma)$ is the "norm" 
of $\gamma$ ). Then, if the fixed points of $\gamma$ are $z_{1}$ and $z_{2}$, then $C(\gamma)=\{\sigma \in \Gamma$ : $\left.\sigma z_{1}=z_{1}, \sigma z_{2}=z_{2}\right\}$. This is an infinite cyclic group. Let $\gamma_{0}$ be the generator of $C(\gamma)$ with the property that $\gamma=\gamma_{0}^{l}$ with a positive integer $l$. Then $h^{-1} C(\gamma) h$ is the group generated by the dilation $z \rightarrow N\left(\gamma_{0}\right) z$, and a fundamental domain of this group in $H$ is $\left\{z \in H: 1 \leq|z|<N\left(\gamma_{0}\right)\right\}$, so by the substitution $z=r e^{i(\pi / 2+\vartheta)}\left(r \in\left(1, N\left(\gamma_{0}\right)\right), \vartheta \in(-\pi / 2, \pi / 2)\right)$ we deduce (since $\left.d \mu_{z}=\frac{d x d y}{y^{2}}=\frac{r d r d \vartheta}{r^{2} \cos ^{2} \vartheta}\right)$ by (2) that

$$
T_{\gamma}=\int_{-\pi / 2}^{\pi / 2} \int_{1}^{N\left(\gamma_{0}\right)} k\left(\frac{N(\gamma)+N(\gamma)^{-1}-2}{4 \cos ^{2} \vartheta}\right) u\left(h\left(r e^{i(\pi / 2+\vartheta)}\right)\right) \frac{d r d \vartheta}{r \cos ^{2} \vartheta} .
$$

Introduce the notation

$$
F(z)=\int_{1}^{N\left(\gamma_{0}\right)} u(h(r z)) \frac{d r}{r} \quad(z \in H) .
$$

Then

$$
T_{\gamma}=\int_{-\pi / 2}^{\pi / 2} k\left(\frac{N(\gamma)+N(\gamma)^{-1}-2}{4 \cos ^{2} \vartheta}\right) F\left(e^{i(\pi / 2+\vartheta)}\right) \frac{d \vartheta}{\cos ^{2} \vartheta} .
$$

Now, $F$ is constant on euclidean lines through the origin, i.e. $F(z)=F(r z)$ for all $r>0$, because $u(h(z))$ is automorphic with respect to $h^{-1} \Gamma h$. In particular,

$$
u\left(h\left(N\left(\gamma_{0}\right) z\right)\right)=u(h(z)) \text { for } z \in H .
$$

So $F$ depends only on $\vartheta$ (if $z=r e^{i(\pi / 2+\vartheta)}$ ), i.e. $F(z)=F(\vartheta)$, where $F$ is a function on $(-\pi / 2, \pi / 2)$.

On the other hand, since $u$ is an eigenfunction of $\Delta$ with eigenvalue $\lambda$, so is $F(z)$ (because $\Delta$ commutes with the group action). Using the form of the Laplace operator in polar coordinates $\left(\Delta=(r \cos \vartheta)^{2}\left(\partial^{2} / \partial r^{2}+r^{-1} \partial / \partial r+\right.\right.$ $\left.r^{-2} \partial^{2} / \partial \vartheta^{2}\right)$ ), we find that $F(\vartheta)$ satisfies a second order ordinary differential equation, which depends only on $\lambda$ :

$$
F^{(2)}(\vartheta)=\frac{\lambda}{\cos ^{2} \vartheta} F(\vartheta) \quad(\vartheta \in(-\pi / 2, \pi / 2)) .
$$

Let $f_{\lambda}(\vartheta)$ be the solution of this differential equation with $f_{\lambda}(0)=1$, $f_{\lambda}^{(1)}(0)=0$, and $\widetilde{f}_{\lambda}(\vartheta)$ the one with $\widetilde{f}_{\lambda}(0)=0, \widetilde{f}_{\lambda}^{(1)}(0)=1$. Then $F(\vartheta)=$ $F(0) f_{\lambda}(\vartheta)+F^{(1)}(0) \widetilde{f}_{\lambda}(\vartheta)$, and $\widetilde{f}_{\lambda}(\vartheta)$ is an odd function, so it gives 0 in $T_{\gamma}$, i.e.

$$
T_{\gamma}=F(0) \int_{-\pi / 2}^{\pi / 2} k\left(\frac{N(\gamma)+N(\gamma)^{-1}-2}{4 \cos ^{2} \vartheta}\right) f_{\lambda}(\vartheta) \frac{d \vartheta}{\cos ^{2} \vartheta} .
$$

Here $F(0)=\int_{1}^{N\left(\gamma_{0}\right)} u(h(r i)) \frac{d r}{r}=\int_{C_{\gamma}} u d S$, where $d S=|d z| / y$ is the hyper- 
bolic arc length, and $C_{\gamma}$ is the closed geodesic $C_{\gamma}=C(\gamma) \backslash l_{\gamma}$, where $l_{\gamma}$ is the noneuclidean line connecting the fixed points $\left(z_{1}\right.$ and $\left.z_{2}\right)$ of $\gamma$, and we factorize it by the action of $C(\gamma)$ (so we can take for $C_{\gamma}$ any segment of length $\log N\left(\gamma_{0}\right)$ on $\left.l_{\gamma}\right)$. Hence

$$
T_{\gamma}=\left(\int_{C_{\gamma}} u d S\right) \int_{-\pi / 2}^{\pi / 2} k\left(\frac{N(\gamma)+N(\gamma)^{-1}-2}{4 \cos ^{2} \vartheta}\right) f_{\lambda}(\vartheta) \frac{d \vartheta}{\cos ^{2} \vartheta},
$$

when $\gamma$ is hyperbolic.

If $\gamma$ is elliptic, then by conjugation in $\Gamma$ we may assume that its fixed point is a $p \in P$. Then $C(\gamma)=\Gamma_{p}=\{\sigma \in \Gamma: \sigma p=p\}$; this is a finite set, $\left|\Gamma_{p}\right|=m_{p}$. We choose $h \in \mathrm{SL}(2, \mathbb{R})$ such that $h(i)=p$, then $h^{-1} \gamma h=R\left(l \vartheta_{p}\right)$ for some integer $0<l<m_{p}$, where $\vartheta_{p}=\pi / m_{p}$, and

$$
R(\varphi)=\left(\begin{array}{cc}
\cos \varphi & \sin \varphi \\
-\sin \varphi & \cos \varphi
\end{array}\right) \text {. }
$$

Then, by (2),

$$
T_{\gamma}=\frac{1}{m_{p}} \int_{H} k\left(z, R\left(l \vartheta_{p}\right) z\right) u(h z) d \mu_{z} .
$$

We use the substitution $z=R(\varphi) e^{-r} i$, i.e. we use geodesic polar coordinates (see [I], Sections 1.3 and 10.6), where $r \in(0, \infty), \varphi \in(0, \pi)$, getting

$$
T_{\gamma}=\frac{1}{m_{p}} \int_{0}^{\infty} k\left(\sin ^{2} l \vartheta_{p} \sinh ^{2} r\right)\left(\int_{0}^{\pi} u\left(h\left(R(\varphi) e^{-r} i\right)\right) d \varphi\right)(2 \sinh r) d r
$$

because $R(\varphi)$ commutes with $R\left(l \vartheta_{p}\right)$, and with $z=e^{-r} i$ we have

$$
\frac{\left|z-R\left(l \vartheta_{p}\right) z\right|^{2}}{4 \operatorname{Im} z \operatorname{Im} R\left(l \vartheta_{p}\right) z}=\sin ^{2} l \vartheta_{p} \sinh ^{2} r,
$$

and furthermore $d \mu_{z}=(2 \sinh r) d r d \varphi$. Define

$$
G(z)=\frac{1}{\pi} \int_{0}^{\pi} u(h(R(\varphi) z)) d \varphi .
$$

One obtains $G(z)$ by averaging the function $u(h(z))$ over the stability group of $i$ in $\operatorname{SL}(2, \mathbb{R})$ (or what amounts to the same, by averaging over noneuclidean circles around $i$ ), so $G(z)$ is radial at $i$, i.e. it depends only on the noneuclidean distance of $z$ and $i$ (see [I], Lemma 1.10). On the other hand, since $u$ is an eigenfunction of $\Delta$ with eigenvalue $\lambda$, so is $G(z)$ (because $\Delta$ commutes with the group action). A radial (at $i$ ) eigenfunction of $\Delta$ of eigenvalue $\lambda$ is determined up to a constant factor ([I], Lemma 1.12), so using the form of the Laplace operator in geodesic polar coordinates $\left(\Delta=\partial^{2} / \partial r^{2}+(\cosh r / \sinh r) \partial / \partial r+(2 \sinh r)^{-2} \partial^{2} / \partial \varphi^{2}\right.$, see [I], (1.20)), we 
find that if $g_{\lambda}(r)(r \in[0, \infty))$ is the unique solution of

$$
g^{(2)}(r)+\frac{\cosh r}{\sinh r} g^{(1)}(r)=\lambda g(r)
$$

with $g_{\lambda}(0)=1$, then $G(z)=u(p) g_{\lambda}(r)$ for $z=R(\varphi) e^{-r} i$, since $h(i)=p$. This shows

$$
T_{\gamma}=\frac{2 \pi}{m_{p}} u(p) \int_{0}^{\infty} k\left(\sin ^{2} l \vartheta_{p} \sinh ^{2} r\right) g_{\lambda}(r) \sinh r d r
$$

when $\gamma$ is elliptic.

If $\gamma$ is parabolic, then by conjugation in $\Gamma$ we may assume that its fixed point is an $a \in A$. Then $C(\gamma)=\Gamma_{a}=\{\sigma \in \Gamma: \sigma a=a\}$. Let $\gamma_{a}$ be a generator of $\Gamma_{a}$. Then $\gamma=\gamma_{a}^{l}$ for some $l \neq 0$. In this case we choose $h=\sigma_{a}$, the scaling matrix, i.e. $\sigma_{a} \infty=a, \sigma_{a}^{-1} \gamma_{a} \sigma_{a}=\left(\begin{array}{ll}1 & 1 \\ 0 & 1\end{array}\right)$. Then by (1) we have

$$
T_{\gamma}^{Y}=\int_{B \backslash\left(\sigma_{a}^{-1} H(Y)\right)} k(z, z+l) u\left(\sigma_{a} z\right) d \mu_{z}
$$

where $B$ is the set of integer translations.

Lemma 1. There is a constant $c_{\Gamma}$ such that

$$
\left\{z \in H: c_{\Gamma} / Y \leq \operatorname{Im} z \leq Y\right\} \subseteq \sigma_{a}^{-1} H(Y) \subseteq\{z \in H: \operatorname{Im} z \leq Y\} .
$$

Proof. If $z \in\left(\sigma_{a}^{-1} H(Y)\right) \cap P(Y)$, then $\gamma \sigma_{a} z \in F(Y) \subseteq F$ for some $\gamma \in \Gamma$ and $\sigma_{a} z \in F_{a}(Y) \subseteq F$, so $\gamma \sigma_{a} z=\sigma_{a} z \in F(Y) \cap F_{a}(Y)=\emptyset$, a contradiction. This proves one half of the lemma, because $\sigma_{a}^{-1} H(Y)$ is invariant under $B=\sigma_{a}^{-1} \Gamma_{a} \sigma_{a}$.

If $z \notin \sigma_{a}^{-1} H(Y)$, then $\operatorname{Im} \sigma_{b}^{-1} \gamma \sigma_{a} z>Y$ for some $\gamma \in \Gamma$ and $b \in A$. Let $\sigma_{b}^{-1} \gamma \sigma_{a}=\left(\begin{array}{cc}\dot{C} & \dot{D}\end{array}\right)$. Then either $C=0$, and in this case $\sigma_{b}^{-1} \gamma \sigma_{a} \infty=$ $\infty$, so $a=b, \sigma_{a}^{-1} \gamma \sigma_{a} \in \sigma_{a}^{-1} \Gamma_{a} \sigma_{a}=B$, and $\operatorname{Im} z>Y$, or $C \neq 0$, when $\operatorname{Im} \sigma_{b}^{-1} \gamma \sigma_{a} z=\operatorname{Im} z /|C z+D|^{2} \leq 1 /\left(C^{2} \operatorname{Im} z\right)$, which means $\operatorname{Im} z<1 /\left(C^{2} Y\right)$. This proves the lemma, because $\min \left\{C>0:\left({ }_{C} \cdot\right) \in \sigma_{b}^{-1} \Gamma \sigma_{a}\right\}$ exists (see [I], pp. 53-54), $a, b \in A, A$ is finite.

This shows that for $\gamma$ parabolic and $Y$ large enough we can integrate in (5) over $\{z=x+i y: 0 \leq y \leq Y, 0 \leq x \leq 1\}$, because $k(z, z+l)=$ $k\left(l^{2} /\left(4 y^{2}\right)\right)$, and this is 0 for $y$ small, since $l \neq 0$, and $k$ has compact support. So

$$
T_{\gamma}^{Y}=\int_{0}^{Y} k\left(\frac{l^{2}}{4 y^{2}}\right)\left(\beta_{a}(0) y^{s}+\widetilde{\beta}_{a}(0) y^{1-s}\right) \frac{d y}{y^{2}},
$$

and with the substitution $\nu=l^{2} /\left(4 y^{2}\right)$ this is

$$
|l|^{-1} \int_{l^{2} /\left(4 Y^{2}\right)}^{\infty} k(\nu)\left(\beta_{a}(0)\left(|l| /\left(2 \nu^{1 / 2}\right)\right)^{s}+\widetilde{\beta}_{a}(0)\left(|l| /\left(2 \nu^{1 / 2}\right)\right)^{1-s}\right) \nu^{-1 / 2} d \nu .
$$


Lemma 2. If $0<\operatorname{Re} S<1$, then, as $Y \rightarrow \infty$,

$$
\begin{aligned}
& \sum_{l \neq 0}|l|^{S-1} \int_{l^{2} /\left(4 Y^{2}\right)}^{\infty} k(\nu)(4 \nu)^{-S / 2} \nu^{-1 / 2} d \nu \\
& =g(0) \frac{Y^{S}}{S}+2^{1-S} \zeta(1-S) \int_{0}^{\infty} k(\nu) \nu^{-(1+S) / 2} d \nu+O\left(Y^{\operatorname{Re} S-1} \log Y\right),
\end{aligned}
$$

where $\zeta$ is the Riemann zeta-function.

P r o of. Summing the left-hand side over $l$ gives

$$
2 \int_{1 /\left(4 Y^{2}\right)}^{\infty} k(\nu)(4 \nu)^{-S / 2} \nu^{-1 / 2}\left(\sum_{1 \leq l \leq 2 Y \sqrt{\nu}} l^{S-1}\right) d \nu .
$$

Since

$$
\sum_{1 \leq l \leq 2 Y \sqrt{\nu}} l^{S-1}=(2 Y \sqrt{\nu})^{S} / S+\zeta(1-S)+O\left((Y \sqrt{\nu})^{\operatorname{Re} S-1}\right),
$$

the lemma follows, because $k$ has compact support, $k(0)$ is finite, $k$ is continuous at 0 , and $2 \int_{0}^{\infty} k(\nu) \nu^{-1 / 2} d \nu=g(0)$.

Summing over the parabolic conjugacy classes means summing over $l \neq 0$ and $a \in A$, so by the above lemma we have proved the following.

Lemma 3. Define

$$
T_{\mathrm{par}}^{Y}=\sum_{\substack{\delta \in \Gamma \\ \delta \text { parabolic }}} \int_{F(Y)} k(z, \delta z) u(z) d \mu_{z} .
$$

Then the difference of $T_{\mathrm{par}}^{Y}$ and

$$
g(0)\left(\frac{Y^{s}}{s} B_{u}+\frac{Y^{1-s}}{1-s} \widetilde{B}_{u}\right)
$$

tends to

$$
B_{u} 2^{1-s} \zeta(1-s) \int_{0}^{\infty} k(\nu) \nu^{-(1+s) / 2} d \nu+\widetilde{B}_{u} 2^{s} \zeta(s) \int_{0}^{\infty} k(\nu) \nu^{(s-2) / 2} d \nu
$$

as $Y \rightarrow \infty$.

4. The spectral trace - end of the proof of Theorem 1. We now compute $\operatorname{Tr}_{u}^{Y} K$ in another way, based on the spectral theorem. Remember that

$$
\operatorname{Tr}_{u}^{Y} K=\sum_{j} h\left(t_{j}\right) I_{u}^{Y}\left(u_{j}\right)+\frac{1}{4 \pi} \int_{-\infty}^{\infty} h(r) I_{u}^{Y}(r) d r
$$


We need several lemmas. Firstly we give the proof of the lemma stated in Section 2.

Proof of Lemma 4. We get the main term in $I_{u}^{Y}(r)$ if we substitute the constant terms of $E_{c}(z, 1 / 2+i r)$ and of $u(z)$ in the cuspidal zones $F_{a}\left(Y_{\Gamma}\right)$; this gives the sum of

$$
\begin{aligned}
& \sum_{a} \sum_{c}\left(\delta_{a c}+\left|\varphi_{a, c}\left(\frac{1}{2}+i r\right)\right|^{2}\right)\left(\beta_{a}(0) \frac{Y^{s}}{s}+\widetilde{\beta}_{a}(0) \frac{Y^{1-s}}{1-s}\right), \\
& \sum_{a} \sum_{c} \delta_{a c} \varphi_{a, c}\left(\frac{1}{2}+i r\right) \\
& \left(\beta_{a}(0) \frac{Y^{s+2 i r}}{s+2 i r}+\widetilde{\beta}_{a}(0) \frac{Y^{1-s+2 i r}}{1-s+2 i r}\right)
\end{aligned}
$$

and

$$
\sum_{a} \sum_{c} \delta_{a c} \varphi_{a, c}\left(\frac{1}{2}+i r\right)\left(\beta_{a}(0) \frac{Y^{s-2 i r}}{s-2 i r}+\widetilde{\beta}_{a}(0) \frac{Y^{1-s-2 i r}}{1-s-2 i r}\right) .
$$

Using $\sum_{c}\left|\varphi_{a, c}(1 / 2+i r)\right|^{2}=1\left([\mathrm{I}]\right.$, Theorem 6.6) and $\overline{\varphi_{a, a}(1 / 2+i r)}=$ $\varphi_{a, a}(1 / 2-i r)$, we find that this main term will be $I_{u}^{Y}(r)-\widetilde{I}_{u}^{Y}(r)$. Similarly, the main term of $I_{u}^{Y}\left(u_{j}\right)$ will be $I_{u}^{Y}\left(u_{j}\right)-\widetilde{I}_{u}^{Y}\left(u_{j}\right)$. Applying Lemmas 5 and 6 below we get the result.

We need the following crude bound.

Lemma 5. For $R \geq 1$ and $Y>0$ we have

$$
\int_{-R}^{R} \sum_{c} \int_{F(Y)}\left|E_{c}(z, 1 / 2+i r)\right|^{2} d \mu_{z} d r=O\left(R^{2}(1+\log Y)\right) .
$$

Proof. This follows easily from [I], formulas (10.9), (6.24) and (10.13).

Lemma 6. For $R \geq 1$ we have

$$
\begin{array}{r}
\int_{-R}^{R}\left(\int_{Y_{\Gamma}}^{\infty} \int_{0}^{1}\left|E_{c}\left(\sigma_{a} z, 1 / 2+i r\right)-\delta_{a c} y^{1 / 2+i r}-\varphi_{a, c}(1 / 2+i r) y^{1 / 2-i r}\right|^{2} y^{\operatorname{Re} s} \frac{d x d y}{y^{2}}\right) d R \\
=O\left(R^{2}\right),
\end{array}
$$

and

$$
\sum_{\left|t_{j}\right| \leq R}\left(\int_{Y_{\Gamma}}^{\infty} \int_{0}^{1}\left|u_{j}\left(\sigma_{a} z\right)-\beta_{a, j}(0) y^{1-s_{j}}\right|^{2} y^{\operatorname{Re} s} \frac{d x d y}{y^{2}}\right)=O\left(R^{2}\right) .
$$

Proof. This follows easily by Parseval's identity, Lemma 7 below, and [I], (8.27) (see also (8.4) and (8.5) there). (We use the Fourier expansions, fix an $n \neq 0$ and sum over the spectrum.) 
LEMMA 7. If $T$ is real and $n \neq 0$ is an integer, then

$$
\begin{aligned}
& \int_{Y_{\Gamma}}^{\infty} \mid\left. W_{1 / 2+i T}(\text { iny })\right|^{2} y^{\operatorname{Re} s} \frac{d y}{y^{2}} \\
& =\left\{\begin{array}{l}
O\left((|T| /|n|)^{\operatorname{Re} s-1} e^{-\pi|T|}\right) \quad \text { if }|n|=O(|T|), \\
\left.O\left(e^{-c_{\Gamma}|n|}\right) \quad \text { if }|T| /|n| \text { is sufficiently small (depending on } \Gamma\right),
\end{array}\right.
\end{aligned}
$$

where $c_{\Gamma}$ is a positive constant depending on $\Gamma$.

Proof. By the definition of $W$ we have

$$
\int_{Y_{\Gamma}}^{\infty}\left|W_{1 / 2+i T}(i n y)\right|^{2} y^{\operatorname{Re} s} \frac{d y}{y^{2}}=O\left(|n|^{1-\operatorname{Re} s} \int_{2 \pi|n| Y_{\Gamma}}\left|K_{i T}(y)\right|^{2} y^{\operatorname{Re} s} \frac{d y}{y}\right),
$$

and the lemma follows by [I], p. 228, the formula above (B.37), and [Le], (5.10.24).

We need one more lemma for the computation of $\operatorname{Tr}_{u}^{Y} K$.

Lemma 8. Let $0<\operatorname{Re} S<1$ and $S \neq 2 s_{l}-1$ for $l \in L$. Then the difference of

$$
\int_{-\infty}^{\infty} h(r) \varphi_{a, a}(1 / 2+i r) \frac{Y^{S-2 i r}}{S-2 i r} d r
$$

and

$$
\pi \varphi_{a, a}\left(\frac{1+S}{2}\right) h\left(i \frac{S}{2}\right)-2 \pi \sum_{\substack{1 / 2<s_{l} \leq(1+\operatorname{Re} S) / 2 \\ l \in L}} h\left(i\left(s_{l}-\frac{1}{2}\right)\right) \frac{Y^{1+S-2 s_{l}}}{1+S-2 s_{l}} R_{a, s_{l}}
$$

tends to 0 as $Y \rightarrow \infty$.

Proof. This follows by replacing the line of integration to $\operatorname{Im} r=$ - Re $S / 2-\varepsilon$ with some $\varepsilon>0$, passing through simple poles at $r=-i S / 2$, and $r=-i\left(s_{l}-1 / 2\right)$ lying in this strip for $l \in L$.

This means that if $h$ satisfies condition (B), then with the notations $\Sigma_{u, h}^{Y}(S)$

$$
=\sum_{1 / 2<s_{l} \leq(1+\operatorname{Re} S) / 2} h\left(t_{l}\right) \frac{Y^{1+S-2 s_{l}}}{1+S-2 s_{l}} \sum_{a} \beta_{a}(0)\left(\sum_{\substack{j \\ s_{j}=s_{l}}}\left|\beta_{a, j}(0)\right|^{2}-R_{a, s_{l}}\right),
$$

$\tilde{\Sigma}_{u, h}^{Y}(S)$

$$
=\sum_{1 / 2<s_{l} \leq(1+\operatorname{Re} S) / 2} h\left(t_{l}\right) \frac{Y^{1+S-2 s_{l}}}{1+S-2 s_{l}} \sum_{a} \widetilde{\beta}_{a}(0)\left(\sum_{\substack{j \\ s_{j}=s_{l}}}\left|\beta_{a, j}(0)\right|^{2}-R_{a, s_{l}}\right),
$$


where $t_{l}=i\left(s_{l}-1 / 2\right)$, we have proved by (6), Lemmas 4 and 8 , using

$$
\frac{1}{2 \pi} \int_{-\infty}^{\infty} h(r) d r=g(0)
$$

the following:

Lemma 9. The difference of $\operatorname{Tr}_{u}^{Y} K$ and

$$
\begin{aligned}
g(0)\left(\frac{Y^{s}}{s} B_{u}+\frac{Y^{1-s}}{1-s} \widetilde{B}_{u}\right)+\frac{1}{2} h\left(i \frac{s}{2}\right) B_{u}(s) \\
\quad+\frac{1}{2} h\left(i \frac{1-s}{2}\right) \widetilde{B}_{u}(1-s)+\Sigma_{u, h}^{Y}(s)+\widetilde{\Sigma}_{u, h}^{Y}(1-s)
\end{aligned}
$$

tends to

$$
\sum_{j} h\left(t_{j}\right) I_{u}\left(u_{j}\right)+\frac{1}{4 \pi} \int_{-\infty}^{\infty} h(r) I_{u}(r) d r
$$

as $Y \rightarrow \infty$.

Since $k$ has compact support, it is obvious that

$$
T_{\text {hyp }}^{Y}=\sum_{\substack{\delta \in \Gamma \\ \delta \text { hyperbolic }}} \int_{F(Y)} k(z, \delta z) u(z) d \mu_{z}
$$

and

$$
T_{\mathrm{ell}}^{Y}=\sum_{\substack{\delta \in \Gamma \\ \delta \text { elliptic }}} \int_{F(Y)} k(z, \delta z) u(z) d \mu_{z}
$$

have finite limits as $Y \rightarrow \infty$ (and of course $T_{\mathrm{id}}^{Y}=\int_{F(Y)} k(z, z) u(z) d \mu_{z}$ tends to 0 as $Y \rightarrow \infty$, because $\int_{F} u(z) d \mu_{z}=0$ ), so by Lemmas 3 and 9 we see that $\Sigma_{u, h}^{Y}(s)+\widetilde{\Sigma}_{u, h}^{Y}(1-s)$ tends to a finite limit as $Y \rightarrow \infty$. But this sum is a finite linear combination of $Y$-powers with nonzero exponents, and every exponent has nonnegative real part. So the fact that this sum has a finite limit as $Y \rightarrow \infty$ implies that this sum is identically 0 . (It is not hard to see that in fact $\sum_{j, s_{j}=s_{l}}\left|\beta_{a, j}(0)\right|^{2}-R_{a, s_{l}}=0$, but we do not need it.)

This last remark, together with (3), (4), Lemmas 3, 4 and 9, proves Theorem 1.

5. The inversion of the integral transformations. The transformation formulas between the functions $k, g$ and $h$ are well known, but we now have a new integral transformation for every $\lambda<0$, namely

$$
R(y)=\int_{-\pi / 2}^{\pi / 2} k\left(\frac{y}{\cos ^{2} \vartheta}\right) f_{\lambda}(\vartheta) \frac{d \vartheta}{\cos ^{2} \vartheta}
$$


for $y>0$. Our aim is now to invert this transformation, i.e. to express $h$ (and in our way $k, q$ and $g$ ) in terms of $R$.

To this end let $R$ be a smooth, compactly supported function on $(0, \infty)$ (i.e. it is 0 in a neighbourhood of 0 as well as in a neighbourhood of $\infty$ ). Denote the Mellin transform of $R$ by

$$
\widehat{R}(s)=\int_{0}^{\infty} R(y) y^{s-1} d y,
$$

and assume that $\widehat{R}(0)=0$ (one needs this unsignificant restriction).

By Mellin inversion

$$
R(y)=\frac{1}{2 \pi i} \int_{(\sigma)} y^{-s} \widehat{R}(s) d s
$$

for $y>0$ and for any real $\sigma$. We see from this that (7) is satisfied with the function $k(\tau)(\tau>0)$ defined by

$$
k(\tau)=\frac{1}{2 \pi i} \int_{(\sigma)} \frac{\tau^{-s}}{F_{\lambda}(s)} \widehat{R}(s) d s,
$$

where $\sigma>1 / 2$, and $F_{\lambda}(s)=\int_{-\pi / 2}^{\pi / 2} f_{\lambda}(\vartheta) \cos ^{2 s-2} \vartheta d \vartheta$. We know $F_{\lambda}(s)$ explicitly,

$$
F_{\lambda}(s)=\sqrt{\pi} \frac{\Gamma\left(s-z_{1}\right) \Gamma\left(s-z_{2}\right)}{\Gamma^{2}(s)},
$$

with $z_{1}=1 / 4+i t / 2, z_{2}=1 / 4-i t / 2,1 / 4+t^{2}=-\lambda$ (see Lemma 11).

It is easy to see from (8) that $k$ is continuous on $[0, \infty), k(0)$ is finite (replace the line of integration by $\sigma=-\varepsilon$ with some $\varepsilon>0$, and use $\widehat{R}(0)=$ 0 ), and $k$ has compact support (this follows from the fact that $R$ has compact support, letting $\sigma \rightarrow \infty$ ), i.e. $k$ satisfies condition (A).

For this $k$ by (8) we get

$$
q(\nu)=\frac{1}{2 \pi i} \int_{0}^{\infty} \frac{\int_{(\sigma)}(\nu+\tau)^{-s} F_{\lambda}^{-1}(s) \widehat{R}(s) d s}{\sqrt{\tau}} d \tau
$$

for $\nu>0$. With the substitution $\tau=\nu \sin ^{2} \vartheta / \cos ^{2} \vartheta, \vartheta \in(0, \pi / 2)$, we have

$$
\int_{0}^{\infty} \frac{(\nu+\tau)^{-s}}{\sqrt{\tau}} d \tau=2 \nu^{1 / 2-s} \int_{0}^{\pi / 2} \cos ^{2 s-2} \vartheta d \vartheta=\nu^{1 / 2-s} E(s),
$$

where $E(s)=\sqrt{\pi} \Gamma(s-1 / 2) / \Gamma(s)$ by the Corollary to Lemma 11. So

$$
q(\nu)=\frac{1}{2 \pi i} \int_{(\sigma)} \nu^{1 / 2-s} \frac{E(s)}{F_{\lambda}(s)} \widehat{R}(s) d s
$$


for $\nu>0$, with

$$
\frac{E(s)}{F_{\lambda}(s)}=\frac{\Gamma(s) \Gamma(s-1 / 2)}{\Gamma\left(s-z_{1}\right) \Gamma\left(s-z_{2}\right)} .
$$

Since $R$ has compact support (as a function on $(0, \infty)$ ), we see by $(9)$ that $q$ is smooth on $(0, \infty), g(\nu)=0$ for $\nu$ large enough (by letting $\sigma \rightarrow \infty$ ), and in a neighbourhood of 0 it has an absolutely convergent expansion of the type $q(\nu)=\sum_{n=0}^{\infty} c_{n} \nu^{n / 2}$ with $c_{1}=0$, i.e. the coefficient of $\nu^{1 / 2}$ is 0 (we see this by letting $\sigma \rightarrow-\infty$, and using $\widehat{R}(0)=0$ ). This implies that the function $g$ (which is even and defined on $(-\infty, \infty)$ ) is smooth on $[0, \infty)$, $g(a)=0$ for $a$ large enough, and for small positive $a$ it has an absolutely convergent expansion of the type $g(a)=\sum_{n=0}^{\infty} d_{n} a^{n}$ with $d_{1}=0$. These properties of $g$ imply (after three-fold integration by parts) that $h$ satisfies condition (B).

Now, let $|\operatorname{Im} r|<1 / 2$. Then by (9), taking $1 / 2+|\operatorname{Im} r|<\sigma<1$ (since the double integral is absolutely convergent in this case) we have

$$
h(r)=\frac{1}{2 \pi i} \int_{(\sigma)} 2^{2 s} \frac{E(s)}{F_{\lambda}(s)} \widehat{R}(s)\left(\int_{-\infty}^{\infty}\left(e^{a}+e^{-a}-2\right)^{1 / 2-s} e^{i r a} d a\right) d s .
$$

We have to compute the inner integral. With the notations

$$
G(A, B)=\int_{0}^{\infty}\left(e^{a}-1\right)^{A} e^{B a} d a, \quad F(r, s)=G(1-2 s,-1 / 2+s+i r)
$$

one obtains

$$
\int_{-\infty}^{\infty}\left(e^{a}+e^{-a}-2\right)^{1 / 2-s} e^{i r a} d a=F(r, s)+F(-r, s) .
$$

By Lemma 10 one has

$$
\begin{aligned}
& F(r, s) \\
& =\pi \frac{\Gamma(-1 / 2+s+i r)}{\Gamma(3 / 2-s+i r) \Gamma(2 s-1)}\{\cot \pi(1-2 s)-\cot \pi(1 / 2-s+i r)\} .
\end{aligned}
$$

So we have determined $h$, but for the application of Theorem 1 we also need

$$
\int_{0}^{\infty} k\left(\sin ^{2} \frac{l \pi}{m_{p}} \sinh ^{2} r\right) g_{\lambda}(r) \sinh r d r
$$

By (8) we have

$$
\int_{0}^{\infty} k\left(\sin ^{2} \frac{l \pi}{m_{p}} \sinh ^{2} r\right) g_{\lambda}(r) \sinh r d r=\frac{1}{2 \pi i} \int_{(\sigma)} \frac{G_{\lambda}(s)}{F_{\lambda}(s)} \widehat{R}(s) \sin ^{-2 s} \frac{l \pi}{m_{p}} d s
$$


for $1 / 2<\sigma<1$, where $G_{\lambda}(s)=\int_{0}^{\infty} g_{\lambda}(r) \sinh ^{1-2 s} r d r$. So by Lemma 11 we obtain

$$
\begin{aligned}
\int_{0}^{\infty} k\left(\sin ^{2} \frac{l \pi}{m_{p}} \sinh ^{2} r\right) g_{\lambda}(r) \sinh r d r \\
=\frac{1}{4 i \sqrt{\pi}} \cdot \frac{1}{\Gamma\left(1-z_{1}\right) \Gamma\left(1-z_{2}\right)} \int_{(\sigma)} \frac{\widehat{R}(s)}{\sin \pi s} \sin ^{-2 s} \frac{l \pi}{m_{p}} d s
\end{aligned}
$$

where $z_{1}=1 / 4+i t / 2, z_{2}=1 / 4-i t / 2,1 / 4+t^{2}=-\lambda$.

We have proved the following.

THEOREM 2. Let $R$ be a smooth, compactly supported function on $(0, \infty)$ (i.e. it is 0 in a neighbourhood of 0 as well as in a neighbourhood of $\infty$ ). Denote the Mellin transform of $R$ by

$$
\widehat{R}(s)=\int_{0}^{\infty} R(y) y^{s-1} d y,
$$

and assume that $\widehat{R}(0)=0$. Then the function $k$ defined by (8) satisfies condition (A), the corresponding h satisfies condition (B), so Theorem 1 is applicable for them. The function $h(r)$ for $|\operatorname{Im} r|<1 / 2$ is given in (10),

$$
\int_{0}^{\infty} k\left(\sin ^{2} \frac{l \pi}{m_{p}} \sinh ^{2} r\right) g_{\lambda}(r) \sinh r d r
$$

is given in (13) (for the functions $E, F_{\lambda}$, and $G_{\lambda}$ see Lemma 11 and its Corollary), and for $y>0$ we have

$$
R(y)=\int_{-\pi / 2}^{\pi / 2} k\left(\frac{y}{\cos ^{2} \vartheta}\right) f_{\lambda}(\vartheta) \frac{d \vartheta}{\cos ^{2} \vartheta} .
$$

\section{Two lemmas on special functions}

LEMma 10. Let

$$
G(A, B)=\int_{0}^{\infty}\left(e^{a}-1\right)^{A} e^{B a} d a \quad \text { for } \operatorname{Re} A>-1, \operatorname{Re}(A+B)<0 .
$$

Then

$$
G(A, B)=\pi \frac{\Gamma(B)}{\Gamma(A+B+1) \Gamma(-A)}\{\cot \pi A-\cot \pi(A+B)\},
$$

where $\cot =\cos / \sin$. 
Proof. We first fix $-1<A<0$, and consider $G(A, B)$ as a function of $B$. In this case we have by partial integration

$$
\begin{aligned}
G(A, B) & =-\int_{0}^{\infty}\left(\frac{\left(e^{a}-1\right)^{A+1}}{A+1}\right)\left(e^{(B-1) a}(B-1)\right) d a \\
& =\frac{1-B}{1+A}(G(A, B)-G(A, B-1)),
\end{aligned}
$$

and this gives

$$
G(A, B)=\frac{B-1}{A+B} G(A, B-1) .
$$

Now let $\widetilde{G}(A, B)=\Gamma(B) / \Gamma(A+B+1)$. Then this satisfies the same functional equation as $G$, i.e.

$$
\widetilde{G}(A, B)=\frac{B-1}{A+B} \widetilde{G}(A, B-1),
$$

so $G(A, B) / \widetilde{G}(A, B)$ (as a function of $B$ ) is periodic with respect to 1 , and it is meromorphic on the whole plane. For $\operatorname{Re} B<-A$ the function $G$ is regular, so in this region the only singularities of $G / \widetilde{G}$ are the roots of $\widetilde{G}$, i.e. $B=-A-1,-A-2, \ldots$ Now, it is easy to see from the integral representation that $G(A, B)$ has a pole of order 1 with residue -1 at $B=$ $-A$, and $\widetilde{G}(A,-A)=\Gamma(-A)$. From these considerations it follows that

$$
\frac{G(A, B)}{\widetilde{G}(A, B)}+\frac{\pi}{\Gamma(-A)} \cot \pi(A+B)
$$

is an entire function of $B$, periodic with respect to 1 , it has at most polynomial growth on vertical lines, so it is a constant. Its value at $B=0$ is $\frac{\pi}{\Gamma(-A)} \cot \pi A$. This proves the lemma for $-1<A<0$, and it is enough by analytic continuation.

For $\lambda<0$ let $f_{\lambda}(\vartheta)(\vartheta \in(-\pi / 2, \pi / 2))$ be the solution of the differential equation

$$
f^{(2)}(\vartheta)=\frac{\lambda}{\cos ^{2} \vartheta} f(\vartheta)
$$

with $f_{\lambda}(0)=1, f_{\lambda}^{(1)}(0)=0$; and let $g_{\lambda}(r)(r \in[0, \infty))$ be the solution of

$$
g^{(2)}(r)+\frac{\cosh r}{\sinh r} g^{(1)}(r)=\lambda g(r)
$$

with $g_{\lambda}(0)=1$.

LEMMA 11. Let $\lambda<0$ and

$$
F_{\lambda}(s)=\int_{-\pi / 2}^{\pi / 2} f_{\lambda}(\vartheta) \cos ^{2 s-2} \vartheta d \vartheta \quad \text { for } \quad \operatorname{Re} s>1 / 2,
$$




$$
G_{\lambda}(s)=\int_{0}^{\infty} g_{\lambda}(r) \sinh ^{1-2 s} r d r \quad \text { for } 1 / 2<\operatorname{Re} s<1
$$

Then

$$
\begin{aligned}
F_{\lambda}(s) & =\sqrt{\pi} \frac{\Gamma\left(s-z_{1}\right) \Gamma\left(s-z_{2}\right)}{\Gamma^{2}(s)} \\
G_{\lambda}(s) & =\frac{\Gamma\left(s-z_{1}\right) \Gamma\left(s-z_{2}\right)}{\Gamma^{2}(s)} \cdot \frac{\pi}{2 \sin \pi s} \cdot \frac{1}{\Gamma\left(1-z_{1}\right) \Gamma\left(1-z_{2}\right)},
\end{aligned}
$$

where $z_{1}=1 / 4+i t / 2, z_{2}=1 / 4-i t / 2,1 / 4+t^{2}=-\lambda$.

Proof. It is easy to see by elementary considerations (using the fact that $\lambda / \cos ^{2} \vartheta$ is negative and it is decreasing for $\left.\vartheta \geq 0\right)$ that $\left|f_{\lambda}(\vartheta)\right| \leq 1$ for every $\vartheta$, and this implies that $f_{\lambda}^{(1)}(\vartheta) \cos \vartheta$ is bounded for a fixed $\lambda$ (since $f_{\lambda}^{(2)}(\vartheta) \cos ^{2} \vartheta$ is bounded).

On the other hand, the function $g_{\lambda}(r)$ is also bounded for a fixed $\lambda$ (for example because there are nonzero bounded eigenfunctions of the Laplace operator on $H$ with eigenvalue $\lambda$ (e.g. $f(z)=f_{\lambda}(\vartheta)$ for $z=r e^{i(\pi / 2+\vartheta)}$ ), and we know ([I], Cor 1.13) that averaging any eigenfunction over hyperbolic circles around any point $w$ in $H$, we get a multiple of $g_{\lambda}(r(z, w))$, where $r$ is the hyperbolic distance), and then $g_{\lambda}^{(1)}(r)$ is also bounded, because $\left(g_{\lambda}^{(1)}(r) \sinh r\right)^{(1)}=\lambda g_{\lambda}(r) \sinh r$. Observe also that $g_{\lambda}^{(1)}(0)=0$. We will repeatedly use these remarks in the following calculations.

Using the differential equation for $f_{\lambda}(\vartheta)$ and partial integration twice we have

$$
\begin{aligned}
\lambda F_{\lambda}(s) & =\int_{-\pi / 2}^{\pi / 2} f_{\lambda}^{(2)}(\vartheta) \cos ^{2 s} \vartheta d \vartheta \\
& =\int_{-\pi / 2}^{\pi / 2} f_{\lambda}(\vartheta)\left[2 s(2 s-1) \cos ^{2 s-2} \vartheta \sin ^{2} \vartheta-2 s \cos ^{2 s} \vartheta\right] d \vartheta
\end{aligned}
$$

and this implies $\lambda F_{\lambda}(s)=2 s(2 s-1) F_{\lambda}(s)-(2 s)^{2} F_{\lambda}(s+1)$, i.e. $F_{\lambda}$ is a meromorphic function on the whole plane satisfying

$$
F_{\lambda}(s+1)=F_{\lambda}(s) \frac{2 s(2 s-1)-\lambda}{(2 s)^{2}} .
$$

Using the differential equation for $g_{\lambda}(r)$ and partial integration we have

$$
\lambda G_{\lambda}(s)=\int_{0}^{\infty}\left(g_{\lambda}^{(1)}(r) \sinh r\right)^{(1)} \sinh ^{-2 s} r d r=2 s \int_{0}^{\infty} g_{\lambda}^{(1)}(r) \sinh ^{-2 s} r \cosh r d r
$$


and a new partial integration gives, by the equality $\cosh ^{2}=1+\sinh ^{2}$, that

$$
\begin{aligned}
\lambda G_{\lambda}(s)= & 2 s(2 s-1) G_{\lambda}(s) \\
& +2 s \lim _{\varepsilon \rightarrow 0+0}\left(2 s \int_{\varepsilon}^{\infty} g_{\lambda}(r) \sinh ^{-2 s-1} r d r-g_{\lambda}(\varepsilon) \sinh ^{-2 s} \varepsilon \cosh \varepsilon\right) .
\end{aligned}
$$

Now, $g_{\lambda}(\varepsilon)=1+O\left(\varepsilon^{2}\right)=\cosh \varepsilon$, from which it follows easily that this last limit is a regular function of $s$ for $-1 / 2<\operatorname{Re} s<1$ and it equals $2 s G_{\lambda}(s+1)$ for $-1 / 2<\operatorname{Re} s<0$. This shows that $G_{\lambda}$ is a meromorphic function on the whole plane satisfying

$$
G_{\lambda}(s+1)=-G_{\lambda}(s) \frac{2 s(2 s-1)-\lambda}{(2 s)^{2}} .
$$

and we also see that in $1 / 2<\operatorname{Re} s<2$ the only pole of $G_{\lambda}(s)$ is at $s=1$, it is of first order and the residue is (from the integral representation) $-1 / 2$.

Let $X_{\lambda}(s)=\Gamma\left(s-z_{1}\right) \Gamma\left(s-z_{2}\right) / \Gamma^{2}(s)$. Then $F_{\lambda} / X_{\lambda}$ is periodic with respect to 1 , and it is regular for $\operatorname{Re} s>1 / 2$, i.e. it is an entire function, and it has at most polynomial growth on vertical lines, so it is a constant. As $s \rightarrow \infty$, we see by Stirling's formula and by $f_{\lambda}(0)=1$ that this constant is $\lim _{s \rightarrow \infty} \sqrt{s} \int_{-\pi / 2}^{\pi / 2} \cos ^{2 s-2} \vartheta d \vartheta$, so it is independent of $\lambda$. For $\lambda \rightarrow 0-0$ we have $X_{\lambda}(s) \rightarrow \Gamma(s-1 / 2) / \Gamma(s)$ and $f_{\lambda}(\vartheta) \rightarrow 1$ for every $\vartheta$, so

$$
\frac{F_{\lambda}}{X_{\lambda}}(1) \rightarrow \frac{\pi}{\Gamma(1 / 2)}=\sqrt{\pi} \text {. }
$$

On the other hand, for

$$
Q_{\lambda}(s)=\frac{G_{\lambda}(s)}{X_{\lambda}(s)}-\frac{\pi}{2 \sin \pi s} \cdot \frac{1}{\Gamma\left(1-z_{1}\right) \Gamma\left(1-z_{2}\right)}
$$

we have $Q_{\lambda}(s+1)=-Q_{\lambda}(s)$, and $Q_{\lambda}$ is regular for $1 / 2<\operatorname{Re} s<2$ (including $s=1$ ), so it is an entire function, it has at most polynomial growth on vertical lines, hence it is identically 0 .

Corollary. For $\operatorname{Re} s>1 / 2$ let $E(s)=\int_{-\pi / 2}^{\pi / 2} \cos ^{2 s-2} \vartheta d \vartheta$. Then

$$
E(s)=\sqrt{\pi} \frac{\Gamma(s-1 / 2)}{\Gamma(s)} .
$$

Pr o o f. This follows by letting $\lambda \rightarrow 0-0$.

\section{References}

[I] H. Iwaniec, Introduction to the Spectral Theory of Automorphic Forms, Rev. Mat. Iberoamericana, 1995.

[Le] N. N. Lebedev, Special Functions and their Applications, 2nd ed., Moscow, Leningrad, 1963. 
[Se] A. Selberg, Harmonic analysis and discontinuous groups in weakly symmetric Riemannian spaces with applications to Dirichlet series, J. Indian Math. Soc. 20 (1956), 47-87.

Mathematical Institute

of the Hungarian Academy of Sciences

Realtanoda u. 13-15

H-1053 Budapest, Hungary

E-mail: biroand@math-inst.hu

Received on 15.9.1997

and in revised form on 15.5.1998 